\title{
Multiverse Model: External Universe(s) as Source of Dark Energy
}

\author{
Werner Krause \\ Berlin, Germany \\ Email:w.w.krause@web.de
}

How to cite this paper: Krause, W. (2021) Multiverse Model: External Universe(s) as Source of Dark Energy. Journal of High Energy Physics, Gravitation and Cosmology, 7, 1306-1314.

https://doi.org/10.4236/jhepgc.2021.74080

Received: July 25, 2021

Accepted: September 14, 2021

Published: September 17, 2021

Copyright $\odot 2021$ by author(s) and Scientific Research Publishing Inc. This work is licensed under the Creative Commons Attribution-NonCommercial International License (CC BY-NC 4.0).

http://creativecommons.org/licenses/by-nc/4.0/ (c) (i) (s) Open Access

\begin{abstract}
The Lambda Cold Dark Matter $(\Lambda \mathrm{CDM})$ model is currently the best model to describe the development of the Universe from the Big Bang to the present time. It is composed of six parameters, two of them, Dark Energy (DE) and $\mathrm{CDM}$, with unknown physical explanations. DE, leading to accelerated expansion of the Universe, is considered a scalar field characterized by exerting its force by repulsive gravity. We examined whether DE can be explained as the warping of spacetime in our Universe by external universes as components of a Multiverse or, in other words, as the gravitational pull exerted by other universes. The acceleration, the resultant kinetic energy, $E_{\text {kin }}$, and the cosmological constant, $\Lambda$, were calculated for one to four external universes. The acceleration is approx. $10^{-11} \mathrm{~m} / \mathrm{s}^{2}$, which is in agreement with observations. Its value is dependent upon the numbers and relative positions of external universes. DE density is approx. $10^{-29} \mathrm{~kg} / \mathrm{m}^{3}$ and $\Lambda$ is in the range of $10^{-38} \mathrm{~s}^{-2}$ and $10^{-55} \mathrm{~m}^{-2}$, respectively. Warping of spacetime by external universes as a physical explanation for DE seems feasible and warrants further considerations.
\end{abstract}

\section{Keywords}

Accelerated Expansion of the Universe, Dark Energy, Gravitational Pull, Warping of Spacetime, Multiverse

\section{Introduction}

The standard model of cosmology, $\Lambda \mathrm{CDM}$ (Lambda Cold Dark Matter), is able to explain the status of our Universe starting from the Big Bang via formation of elements, stars and galaxies until present-day cosmological observations. $\Lambda \mathrm{CDM}$ is based on Einstein's General Relativiy (GR) and the field equations derived from GR: 


$$
G_{\mu v}+\Lambda g_{\mu v}=k T_{\mu v}
$$

with $G_{\mu \nu}=$ Einstein tensor, $\Lambda=$ cosmological constant, $g_{\mu \nu}=$ metric tensor, $\kappa=$ Einstein gravitational constant, and $T_{\mu \nu}=$ stress-energy tensor. According to the original version of GR, which did not contain $\Lambda$, the Universe is static, which means neither expanding nor crunching. Einstein revised this notion when it was discovered that the Universe is expanding by introducing $\Lambda$ [1], which he revoked later [2] but which resurrected in 1998 after discovering the accelerating expansion of our Universe [3] [4].

Six parameters are minimally necessary for the $\Lambda \mathrm{CDM}$ model, age of the Universe, scalar spectral index, curvature fluctuation amplitude, reionization optical depth, baryon matter density, Dark Energy (DE) density, and CDM density. Despite huge efforts, the latter two parameters still cannot be explained physically at present. Nevertheless, the $\Lambda$ CDM model has been and still is the most efficient tool to cover cosmological observations. The characterization and check of feasibility of a physical explanation of $\mathrm{DE}$ is the objective of this paper.

$\mathrm{DE}$ has negative pressure and contributes to $T_{\mu \text { p }}$ the stress-energy tensor, leading to accelerated expansion of the Universe. DE is acting like repulsive gravity constituting approx. $68 \%$ of the mass-energy density of the Universe [5]. The remaining $32 \%$ are CDM (28\%) and normal, baryonic matter (4\%).

The focus of this paper is to check the feasibility for the following physical explanation of DE: warping of spacetime in our Universe due to a source or sources located outside of our Universe. The basis for this hypothesis is the existence of one or more other universes in addition to our Universe (Multiverse model). The concept of multiple universes or Multiverses has controversially been discussed by many physicists, dividing them into two groups, the believers such as for example Tegmark [6], Riess [7], and Hawking [8] and the skeptics, for example, Penrose [9] and Mukhanov [10].

As a check of feasibility of the proposed hypothesis, the kinetic energy of our Universe due to the gravitational pull by one or more external universes has been calculated and from the resulting energy density, the two parameters, accelerated expansion and $\Lambda$, have been obtained using Einstein's field equation. Both, acceleration and $\Lambda$, are in agreement with cosmological observations.

\section{Calculations}

In order to simplify the calculations, it is assumed that all universes have a zero curvature, i.e. they are flat, follow the same laws of physics and are similar in size (radius $r=4.40 \times 10^{26} \mathrm{~m}$ ) and mass $\left(m\right.$ [baryonic + dark matter] $=1.01 \times 10^{54}$ $\mathrm{kg})$. A three-dimensional coordinate system, $x, y, z$, is used with its origin $\mathrm{U}_{0}(0$, $0,0)$ at the center of our Universe. Accordingly, any other universes have $x^{-}, y^{-}$, and $z$-coordinates, which are equal to or larger than twice the radius of the Universe, $r$. In the following, all coordinates are provided as multiples of $r$.

The net gravitational pull, $F$, of external universes is calculated according to Newton's law, $F=G m_{1} m_{2} / R_{i}^{2}$ with $G=6.674 \times 10^{-11} \mathrm{~m}^{3} / \mathrm{kg} / \mathrm{s}^{2}, m_{1}=m_{2}=1.45$ 
$\times 10^{53} \mathrm{~kg}$ (baryonic mass) $+8.7 \times 10^{53} \mathrm{~kg}$ (Cold Dark Matter $[\mathrm{CDM}]$ mass $)=1.01$ $\times 10^{54} \mathrm{~kg}$ (total mass). The mass of CDM is obtained from its density of $2.24 \times$ $10^{-27} \mathrm{~kg} / \mathrm{m}^{3}$, as reported by Carmeli [11], and the volume of our Universe $(3.57 \times$ $\left.10^{80} \mathrm{~m}^{3}\right) . R_{i}$ is the vector pointing from the center of our Universe to the center(s) of the external universe(s).

The overall gravitational pull of more than one external universe, $F$, is obtained by additive vector calculation after determination of the individual $x^{-}, y^{-}$, and $z$-components, $F_{p}$ for the individual pull of each external universe. $R_{i}$ is obtained as $R_{i}=\sqrt{\left(x_{i}-a_{i}\right)^{2}+\left(y_{i}-b_{i}\right)^{2}+\left(z_{i}-c_{i}\right)^{2}}$.

The total gravitational pull, $F_{i}$, of any external universe is obtained from the individual components $F_{x}, F_{y}$, and $F_{z}$ with $F_{x}=F_{i} \cos \alpha, F_{y}=F_{i} \cos \beta$, and $F_{z}=F_{i} \cos \gamma$, with $\cos \alpha=\left(x_{i}-a_{i}\right) / R_{i}, \cos \beta=\left(y_{i}-b_{i}\right) / R_{i}$, and $\cos \gamma=\left(z_{i}-c_{i}\right) / R_{i}$. The overall force, $F$, is then calculated individually summing up the $F_{x}, F_{y}$ and $F_{z}$ components of all external universes according to $F=\sqrt{\sum F_{x}^{2}+\sum F_{y}^{2}+\sum F_{z}^{2}}$. The angle between the overall resulting vector, $R$, and the $\mathrm{z}$-axis is $\theta$ and the angle between the projection of $R$ on the $x y$-plane and the $\mathrm{x}$-axis is $\Phi$.

The acceleration of our Universe induced by gravity of external universes $U_{i}$, is calculated from $a=F / m_{1}$. The net velocity of our Universe due to the net gravitational pull by external Universes is obtained as $v(t)=v\left(t_{0}\right)+a t$ with $v\left(t_{0}\right)$ $=0$. For $t, 13$ billion years $\left(4.10 \times 10^{17} \mathrm{~s}\right)$ is used. The numbers obtained for $a$ and $V$ are net values due to the gravitational pull by external universes, not taking into consideration any effects by the Big Bang, which means $v\left(t_{0}\right)$ is set as zero. The distance the Universe has traveled after 13 billion years is calculated from $d$ $=v t$. The relative distance is $d / r$.

The kinetic energy $E_{k i n}$ of our Universe due to the gravitational pull of the external universe(s) is calculated according to $E_{k i n}=1 / 2 m_{1} v^{2}$ with $v=$ the velocity of our Universe due to the gravitational pull after $t=13$ billion years. $E_{k i n}$ is defined as DE. With $E_{k i n}=m c^{2}$, mass, $m$, is calculated and after division with the volume of our Universe $V$, the density of $\mathrm{DE}, \rho_{\lambda}$, is obtained. Einstein's field equations are then used for the calculation of $\Lambda$ according to

$$
G_{\mu v}+\Lambda g_{\mu v}=k T_{\mu v}
$$

With $G_{\mu \nu}=$ Einstein tensor, $\Lambda=$ cosmological constant, $g_{\mu v}=$ metric tensor, $\kappa$ $=$ Einstein gravitational constant, and $T_{\mu \nu}=$ stress-energy tensor leading to

$$
G_{\mu v}+\Lambda g_{\mu v}=8 \pi G T_{\mu v}
$$

and, finally to

$$
\Lambda=8 \pi G \rho_{\lambda},
$$

with $\rho_{\lambda}=$ density of $\mathrm{DE}$, for $\Lambda$ with the dimension $\mathrm{s}^{-2}$ and to

$$
\Lambda=4 \pi G \rho_{\lambda} / c^{2}
$$

with $c=$ speed of light, for $\Lambda$ with the dimension $\mathrm{m}^{-2}$. 


\section{Results}

The force, $F$, on our Universe by the gravitational pull of one external universe $U$ $(2,0,0)$ at a location twice the radius $r$ from the center of our Universe, i.e. directly adjacent, is $8.88 \times 10^{43} \mathrm{~N}$ (Figure 1 , Table 1 ) resulting in a net acceleration of our Universe of $8.75 \times 10^{-11} \mathrm{~m} / \mathrm{s}^{2}$ and a net velocity of $35,865 \mathrm{~km} / \mathrm{s}$. As a consequence, after 13 billion years the Universe has moved $1.47 \times 10^{25} \mathrm{~m}$ or 0.03 $r$ from its original position in the direction of $U_{1}$ and the latter has moved the same distance in the direction of our Universe.

The kinetic energy, $E_{k i m}$, of our Universe due to the gravitational pull by one external universe is $6.53 \times 10^{68} \mathrm{~J}$ from which an energy density, $\rho_{\Lambda}$, of $2.03 \times 10^{-29}$ $\mathrm{kg} / \mathrm{m}^{3}$ is calculated. The cosmological constant, $\Lambda$, is then obtained as $3.41 \times$ $10^{-38} \mathrm{~s}^{-2}$ and $1.90 \times 10^{-55} \mathrm{~m}^{-2}$, respectively.

If the external universe is located further away, e.g. at position $U(10,0,0)$, we get $F=3.55 \times 10^{42} \mathrm{~N}, a=3.50 \times 10^{-12} \mathrm{~m} / \mathrm{s}^{2}$, and $v=1434 \mathrm{~km} / \mathrm{s}$, for $\mathrm{U}(100,0,0)$ we have $F=3.55 \times 10^{40} \mathrm{~N}$ and $v=14.3 \mathrm{~km} / \mathrm{s}$ and for $U(1000,0,0)$ we obtain $F=$ $3.55 \times 10^{38} \mathrm{~N}, a=3.50 \times 10^{-16} \mathrm{~m} / \mathrm{s}^{2}$, and $v=0.14 \mathrm{~km} / \mathrm{s}$. The dependence of $F, a$, $\rho_{\Lambda}$, and $\Lambda$ on the location of an external universe at position $\mathrm{U}(n, 0,0)$ with $n=$ 2 - 10 is illustrated in Figure 2. The density of DE, $\rho_{\lambda}$, is $\rho_{\lambda}=1.64 \times 10^{-29} \mathrm{~kg} / \mathrm{m}^{3}$ for $U(2,0,0)$. For $U(10,0,0)$ we obtain $\rho_{\lambda}=2.62 \times 10^{-32} \mathrm{~kg} / \mathrm{m}^{3}$ and for $\mathrm{U}(100$,

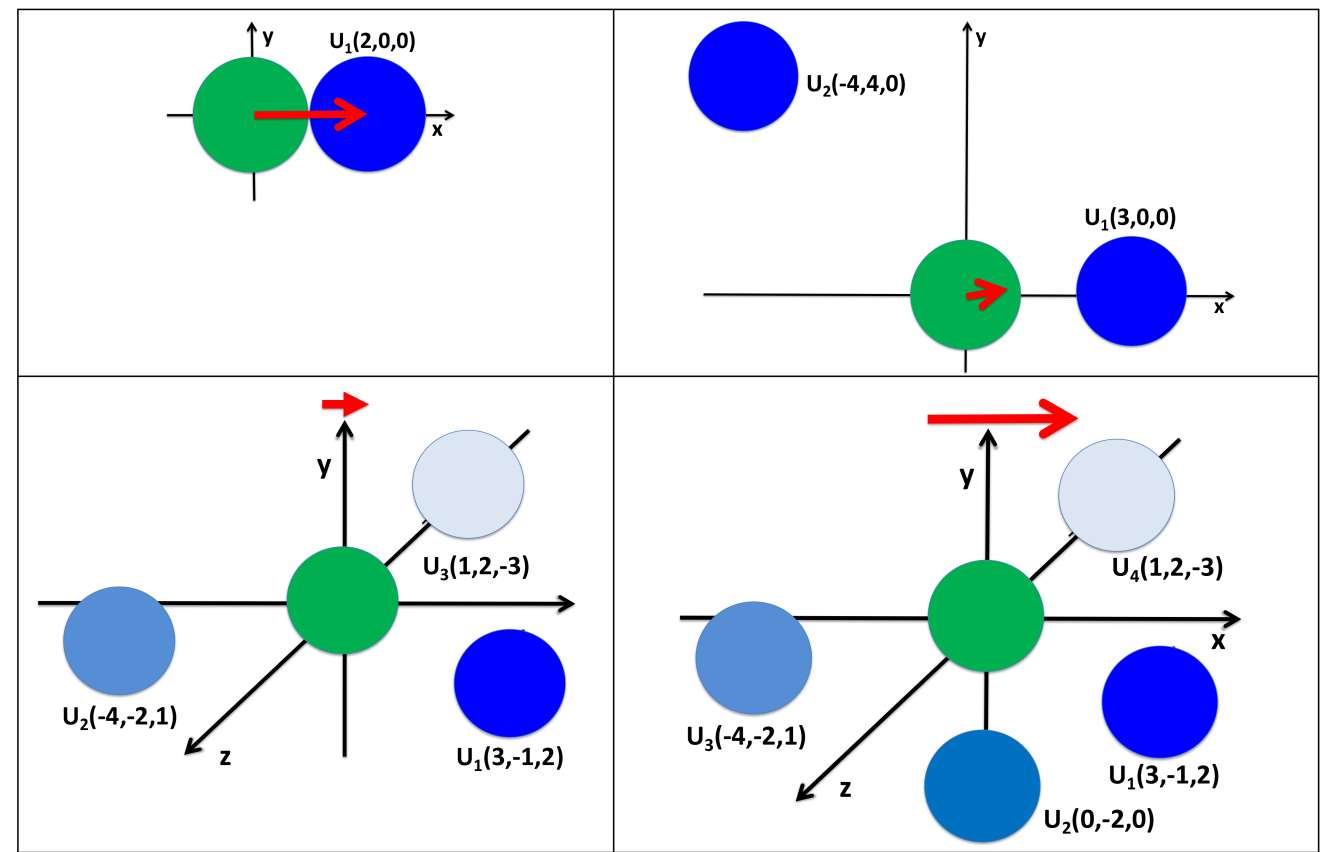

Figure 1. Four models with external universes exerting gravitational pull on our Universe. Top: Two-dimensional examples. Bottom: Three-dimensional versions. The coordinates of the universes are multiples of the radius, $r$. In the two-dimensional graphs, the red arrows represent the direction and the value of the gravitational force $F$. The lengths of the arrows are relative to the gravitational force exerted by the gravitational pull of external universes. In the 3-dimensional graphs, only the relative lengths of the arrows are provided, not their directions. The absolute values of $F$ and the corresponding angles $\Phi$ and $\theta$ are listed in Table 1 . The ranking of the absolute values of $F$ is $F_{4}>F_{1}>$ $F_{2}>F_{3}$. 
Table 1. Comparison of the parameters obtained for four different Mulitverse models with one to four external universes.

\begin{tabular}{ccccc}
\hline Parameter & $\mathrm{U}(2)$ & $\mathrm{U}(3)$ & $\mathrm{U}(4)$ & $\mathrm{U}(5)$ \\
\hline$F(\mathrm{~N})$ & $8.88 \times 10^{43}$ & $3.26 \times 10^{43}$ & $2.30 \times 10^{43}$ & $9.86 \times 10^{43}$ \\
$a\left(\mathrm{~m} / \mathrm{s}^{2}\right)$ & $8.75 \times 10^{-11}$ & $3.21 \times 10^{-11}$ & $2.27 \times 10^{-11}$ & $9.72 \times 10^{-11}$ \\
$V(\mathrm{~km} / \mathrm{s})$ & 35,865 & 13,157 & 9292 & 39,846 \\
$d(\mathrm{~m})$ & $1.47 \times 10^{25}$ & $5.39 \times 10^{24}$ & $3.81 \times 10^{24}$ & $1.63 \times 10^{25}$ \\
$d(\mathrm{r})$ & 0.03 & 0.01 & 0.01 & 0.04 \\
$\Phi\left(^{\circ}\right)$ & 0 & 13.9 & -68.5 & -78.0 \\
$\theta\left(^{\circ}\right)$ & 90 & 90 & 41.4 & 82.1 \\
$E_{k i n}(\mathrm{~J})$ & $6.53 \times 10^{68}$ & $8.79 \times 10^{67}$ & $4.38 \times 10^{67}$ & $8.06 \times 10^{68}$ \\
$\rho_{\Lambda}\left(\mathrm{kg} / \mathrm{m}^{3}\right)$ & $2.03 \times 10^{-29}$ & $2.74 \times 10^{-30}$ & $1.37 \times 10^{-30}$ & $2.51 \times 10^{-29}$ \\
$\Lambda\left(\mathrm{s}^{-2}\right)$ & $3.41 \times 10^{-38}$ & $4.59 \times 10^{-39}$ & $2.29 \times 10^{-39}$ & $4.21 \times 10^{-38}$ \\
$\Lambda\left(\mathrm{m}^{-2}\right)$ & $1.90 \times 10^{-55}$ & $2.56 \times 10^{-56}$ & $1.27 \times 10^{-56}$ & $2.34 \times 10^{-55}$ \\
\hline
\end{tabular}

0, 0) $\rho_{\lambda}=2.62 \times 10^{-36} \mathrm{~kg} / \mathrm{m}^{3}$. The value for the cosmological constant, $\Lambda$, in these three cases is $\Lambda_{\mathrm{U} 2}=2.75 \times 10^{-38} \mathrm{~s}^{-2}$ or $1.53 \times 10^{-55} \mathrm{~m}^{-2}, \Lambda_{\mathrm{U} 10}=4.40 \times 10^{-41} \mathrm{~s}^{-2}$ or $2.45 \times 10^{-58} \mathrm{~m}^{-2}$, and $\Lambda_{\mathrm{U} 100}=4.40 \times 10^{-45} \mathrm{~s}^{-2}$ or $2.45 \times 10^{-62} \mathrm{~m}^{-2}$. Increasing the distance expectedly results in a decrease of force, acceleration, DE density and cosmological constant.

On the other hand, if the mass of the external universe is increased, this also increases $F, a, \rho_{\Lambda}$, and $\Lambda$, as illustrated in Figure 2 for acceleration and $\Lambda$.

In the model with two external universes at positions $U_{1}(3,0,0)$ and $U_{2}(-4$, 4,0 ), we obtain a gravitational pull of $F=3.26 \times 10^{43} \mathrm{~N}$, a net acceleration of $a=$ $3.21 \times 10^{-11} \mathrm{~m} / \mathrm{s}^{2}$, and a net velocity of $v=13,157 \mathrm{~km} / \mathrm{s}$. The DE density is $2.74 \times$ $10^{-30} \mathrm{~kg} / \mathrm{m}^{3}$ and $\Lambda=4.59 \times 10^{-39} \mathrm{~s}^{-2}$ or $2.56 \times 10^{-56} \mathrm{~m}^{-2}$.

In the two three-dimensional graphs, the respective values are $F=2.30 \times 10^{43}$ $\mathrm{N}$ and $F=9.86 \times 10^{43} \mathrm{~N}, a=2.27 \times 10^{-11} \mathrm{~m} / \mathrm{s}^{2}$ and $a=9.72 \times 10^{-11} \mathrm{~m} / \mathrm{s}^{2}$, and $v=$ $9292 \mathrm{~km} / \mathrm{s}$ and $v=39,846 \mathrm{~km} / \mathrm{s}$, respectively. For $\rho_{\lambda}$ we obtain $1.37 \times 10^{-30} \mathrm{~kg} / \mathrm{m}^{3}$ and $2.51 \times 10^{-29} \mathrm{~kg} / \mathrm{m}^{3}$. The cosmological constants are calculated as $2.29 \times 10^{-39}$ $\mathrm{s}^{-2}\left(1.27 \times 10^{-56} \mathrm{~m}^{-2}\right)$ and $4.21 \times 10^{-38} \mathrm{~s}^{-2}\left(2.34 \times 10^{-55} \mathrm{~m}^{-2}\right)$.

\section{Discussion}

Although the number of theories as modifications or alternatives to the $\Lambda \mathrm{CDM}$ model is constantly increasing, the introduction of the Cosmological Constant $\Lambda$ by Einstein in 1917 [1] [2] was the first and still remains the most effective approach to keep the General Relativity equation for large cosmological scales on track. A physical explanation for $\Lambda$ led to the introduction of a scalar field [12], without, however, being able to explain what kind of scalar field this could be. The model was modified later on in various ways [13], including the incorporation of additional dimensions [14] and numerous other approaches without 


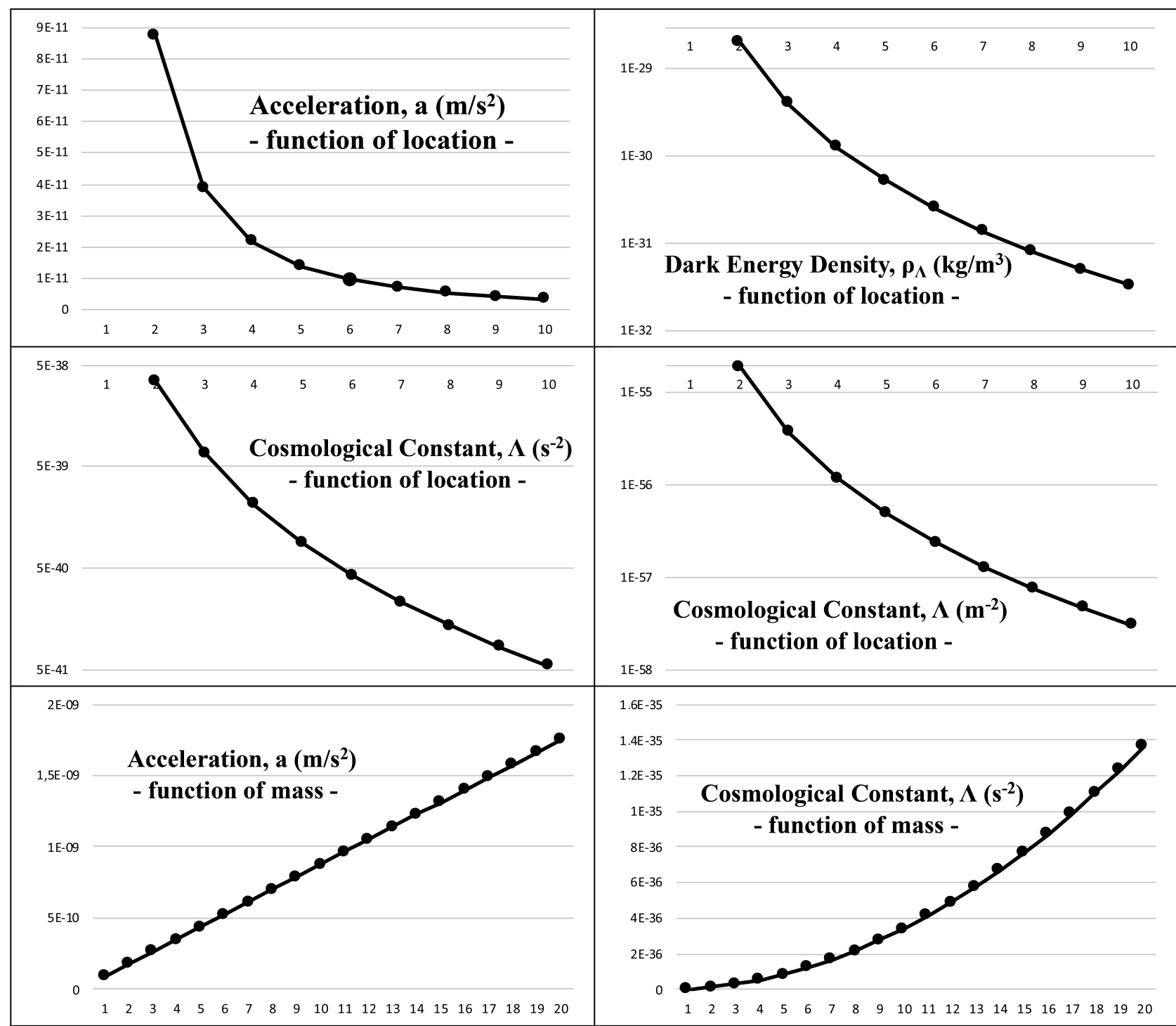

Figure 2. Top Left: Acceleration in $\mathrm{m} / \mathrm{s}^{2}$ of our Universe due to the external pull of one external universe vs. the positions $\mathrm{U}$ ( $n, 0$, 0 ) with $n=2-10$. The coordinates of $\mathrm{U}(n, 0,0)$ are multiples of the radius, $r$. Top Right: Density of Dark Energy, $\rho_{\Lambda}$, in $\mathrm{kg} / \mathrm{m}^{3}$ as a function of the distance $r$ (radius of the universe) of one external universe located at $\mathrm{U}(n, 0,0)$ with $n=2-10$. The $\mathrm{y}$-axis is on a logarithmic scale. $2^{\text {nd }}$ Row Left: Cosmological constant, $\Lambda$, in $\mathrm{s}^{-2}$ depending on the location of one external universe at position $\mathrm{U}$ $(n, 0,0)$ with $n=2-10$. The y-axis is on a logarithmic scale. $2^{\text {nd }}$ Row Right: Cosmological constant, $\Lambda$, in $\mathrm{m}^{-2}$ depending on the location of one external universe at position $\mathrm{U}(n, 0,0)$ with $n=2-10$. The y-axis is on a logarithmic scale. Bottom Left: Acceleration $a$ in $\mathrm{m} / \mathrm{s}^{2}$ depending on the relative mass $n$ of one external universe at position $\mathrm{U}(2,0,0)$ with $n=1-20$ multiples of the mass of the external universe. Bottom Right: Cosmological constant, $\Lambda$, in s ${ }^{-2}$ depending on the relative mass $n$ of one external universe at position $\mathrm{U}(2,0,0)$ with $n=1-20$ multiples of the mass of the external universe.

being able to replace the $\Lambda$ CDM model.

Other models that have widely been discussed are those based on extended gravity, for example, interferometric detection of gravitational waves as a definitive test of GR, as proposed by Corda [15], or in general, extensive evaluation of all aspects of gravitational waves leading to gravitational physics and astronomy, as described by several groups [16]-[21].

The objective of the present paper is based on the idea that repulsive gravity, 
which is considered as the driving force of $\mathrm{DE}$, might easily be substituted by "normal", attractive gravity if the source of this force is placed outside of our Universe. This proposal requires the presence of a Multiverse, which means the existence of more than one universe. Using three-dimensional vector calculation and Newton's Law on Gravity, the gravitational pull of external universes on our Universe was calculated for one, two, three, and four external universes. The total mass taken into account for a universe included baryonic matter and Dark Matter. For calculating the latter mass, DM density data published by Carmeli [11] were used. A recent paper by Kusenko [22] proposes CDM as black holes in a Multiverse.

The acceleration achieved due to the gravitational pull by external universes is 2.3 to $9.7 \times 10^{-11} \mathrm{~m} / \mathrm{s}^{2}$ for all four models. This is in agreement with a 1 $\mathrm{km}^{2} / \mathrm{s}^{2} \mathrm{pc} \approx 3 \times 10^{-11} \mathrm{~m} / \mathrm{s}^{2}$ as reported by Walker [23].

From the acceleration we can calculate the kinetic energy, $E_{k i n}$, induced by the gravitational pull and the density of $E_{k i n}, \rho_{\Lambda}$. The kinetic energy of the Universe due to the acceleration induced by the external universes is proposed as the physical explanation of DE. $E_{k i n}$ is in the range of $4.4 \times 10^{67} \mathrm{~J}$ to $8.1 \times 10^{68} \mathrm{~J}$ and $\rho_{\Lambda}$ is $1.4 \times 10^{-30} \mathrm{~kg} / \mathrm{m}^{3}$ to $2.5 \times 10^{-29} \mathrm{~kg} / \mathrm{m}^{3}$. The cosmological constant, $\Lambda$, was then obtained using Einstein's field equations ranging from $2.3 \times 10^{-39} \mathrm{~s}^{-2}$ or 1.3 $\times 10^{-56} \mathrm{~m}^{-2}$ to $4.2 \times 10^{-38} \mathrm{~s}^{-2}$ or $2.3 \times 10^{-55} \mathrm{~m}^{-2}$, which is somewhat smaller than the $10^{-35} \mathrm{~s}^{-2}$ and $10^{-52} \mathrm{~m}^{-2}$ reported by Carmeli [11] and Aghanim [24] or the $7.23 \times 10^{-36} \mathrm{~s}^{-2}$ published by Farnes [25].

The data provided in the present paper for $E_{k i n}$ and $\Lambda$ have to be considered a first and rough check of the feasibility of this proposal and need further modeling. For example, the kinetic energies provided above do not take into consideration that the universes are approaching each other and therefore the distance $r$ is decreasing, which results in an increase with time of $E_{k i n}$ and, likewise, of the cosmological constant, $\Lambda$. Other factors for modeling include the numbers, locations, and masses of the external universes.

\section{Conclusion}

The data obtained in this paper indicate that the accelerated expansion of our Universe can be explained by defining DE as the warping of spacetime by a source outside of our Universe. The kinetic energy caused by the gravitational pull by one or more external universes provides a direct measure for the warping of spacetime from outside. The concept is based on the assumption that we are living in a Multiverse and that the external universes are identical to ours in terms of physical laws and constants, at least regarding Newton's Law on Gravity.

\section{Conflicts of Interest}

The author declares no conflicts of interest regarding the publication of this paper. 


\section{References}

[1] Einstein, A. (1917) Kosmologische Betrachtungen zur allgemeinen Relativitätstheorie. Sitzungsberichte der Königlich Preußischen Akademie der Wissenschaften, 142.

[2] Einstein, A. (1931) Zum kosmologischen Problem der allgemeinen Relativitätstheorie. Sitzungsber. Preuss. Akad. Wiss. Berlin, 235.

[3] Riess, A.G. (1998) Observational Evidence from Supernovae for an Accelerating Universe and a Cosmological Constant. The Astronomical Journal, 116, 1009.

https://doi.org/10.1086/300499

[4] Perlmutter, S., et al. (1999) The Supernova Cosmology Project, Measurements of Omega and Lambda from 42 High-Redshift Supernovae. The Astrophysical Journal, $517,565$.

[5] Maeder, A., et al. (2020) Planck 2018 Results. VI. Cosmological Parameters. Astronomy \& Astrophysics, 641, A6. https://doi.org/10.1051/0004-6361/202039265

[6] Tegmark, M. (2003) Parallel Universes, Honoring John Wheeler's 90th Birthday. Cambridge University Press, Cambridge. arXiv: astro-ph/0302131.

[7] Riess, A.G., et al. (2001) The Farthest Known Supernova: Support for an Accelerating Universe and a Glimpse of the Epoch of Deceleration. The Astrophysical Journal, 560, 49. https://doi.org/10.1086/322348

[8] Hawking, S.W. and Hertog, T. (2018) A Smooth Exit from Eternal Inflation? Journal of High Energy Physics, 2018, Article No. 147.

https://doi.org/10.1007/JHEP04(2018)147

[9] Penrose, R. (2012) Cycles of Time, an Extraordinary New View of the Universe. Vintage Books, a Division of Random House Incorporated, New York.

[10] Mukhanov, V. (2014) Inflation without Selfreproduction. Fortschritte der Physik, 63, 36-41. https://doi.org/10.1002/prop.201400074

[11] Carmeli, M. and Kuzmenko, T. (2001) Value of the Cosmological Constant: Theory versus Experiment. AIP Conference Proceedings, 586, 316.

https://doi.org/10.1063/1.1419571

[12] Ratra, P. and Peebles, L. (1988) Cosmological Consequences of a Rolling Homogeneous Scalar Field. Physical Review D, 37, 3406.

https://doi.org/10.1103/PhysRevD.37.3406

[13] Carroll, S.M., Duvvuri, V., Trodden, M., et al. (2004) Is Cosmic Speed-Up Due to New Gravitational Physics? Physical Review D, 70, Article ID: 043528.

https://doi.org/10.1103/PhysRevD.70.043528

[14] Dvali, G.R., Gabadadze, G. and Porrati, M. (2000) 4D Gravity on a Brane in 5D Minkowski Space. Physics Letters B, 485, 208-214.

https://doi.org/10.1016/S0370-2693(00)00669-9

[15] Corda, C. (2009) Interferometric Detection of Gravitational Waves: The Definitive Test for General Relativity. International Journal of Modern Physics D, 18, 2275-2282. https://doi.org/10.1142/S0218271809015904

[16] Arimoto, M., et al. (2021) Gravitational Wave Physics and Astronomy in the Nascent Era. Progress of Theoretical and Experimental Physics, ptab042. https://doi.org/10.1093/ptep/ptab042

[17] Ghosh, S., Kastaun, W. and Pratten, G. (2021) Rapid Model Comparison of Equations of State from Gravitational Wave Observation of Binary Neutron Star Coalescences. arXiv: 2104.08681 [gr-qc].

[18] Talbot, C., Thrane, E., Biscoveanu, S. and Smith, R. (2021) Inference with Finite 
Time Series: Observing the Gravitational Universe through Windows. arXiv: 2106.13785 [astro-ph.IM]

[19] Sedda, M.A., et al. (2021) The Missing Link in Gravitational-Wave Astronomy. A Summary of Discoveries Waiting in the Decihertz Range. Experimental Astronomy. https://doi.org/10.1007/s10686-021-09713-Z

[20] Cai, R.-G., Cao, Z., Guo, Z.-K., Wang, S.-J. and Yang, T. (2017) The Gravitational-Wave Physics. National Science Review, 4, 687-706.

[21] Bian, L., et al. (2021) The Gravitational-Wave Physics II: Progress. arXiv: 2106.10235 [gr-qc].

[22] Kusenko, A., et al. (2020) Exploring Primordial Black Holes from the Multiverse with Optical Telescopes. Physical Review Letters, 125, Article ID: 181304. https://doi.org/10.1103/PhysRevLett.125.181304

[23] Walker, M.G. and Loeb, A. (2014) Is the Universe Simpler than LCDM? Contemporary Physics, 55, 198-211. https://doi.org/10.1080/00107514.2014.919741

[24] Aghanim, N., et al. (2019) Planck 2018 Results. VI. Cosmological Parameters Astronomy and Astrophysics Manuscript No. msc ESO 2019.

[25] Farnes, J.S. (2018) A Unifying Theory of Dark Energy and Dark Matter: Negative Masses and Matter Creation within a Modified $\Lambda$ CDM Framework. Astronomy and Astrophysics, 620, A92. https://doi.org/10.1051/0004-6361/201832898 